\title{
Estimativa de Emissões de Gases de Efeito Estufa em Reservatórios a Partir da Dinâmica da Matéria Orgânica na Coluna da Água: Estudo de Caso PCH Salto Natal, Campo Mourão - Paraná
}

\author{
Rita Christianne Sbrissia \\ J. Malucelli Energia S.A. \\ ritacss@jmalucelli.com.br \\ Cristovão Vicente Scapulatempo Fernandes, Maria Cristina Borba Braga \\ Universidade Federal do Paraná, DHS/PPGERHA \\ cris.dhs@ufpr.br,crisbraga@ufpr.br \\ Antonio Fonseca dos Santos \\ Brookfield Energia Renovável \\ antonio.fonseca@brookfieldenergia.com
}

Recebido: 15/10/09 - revisado: 30/05/10 - aceito: 28/07/11

\section{RESUMO}

Na década de 1990, alguns estudos apresentaram evidências indicando que os reservatórios de usinas hidrelétricas seriam emissores potenciais de gases de efeito estufa para a atmosfera. Para justificar novos projetos de usinas hidrelétricas deve-se mostrar, entre outros aspectos, que esta é a melhor solução em termos ambientais para uma dada capacidade instalada. Adicionalmente, é relevante avaliar o potencial de redução de gases de efeito estufa em relação a outras fontes de energia. No entanto, muitas pesquisas realizadas com este objetivo fundamentam seu procedimento metodológico para o monitoramento dos gases de efeito estufa sem considerar os aspectos do ciclo da matéria orgânica no reservatório e sua sinergia com a dinâmica de processos de transporte que compõem uma bacia hidrográfica. Este trabalho apresenta uma metodologia baseada no balanço de carbono do reservatório de uma pequena central hidrelétrica (PCH) para estimar as emissões de gases de efeito estufa, especialmente metano $\left(\mathrm{CH}_{4}\right)$ e dióxido de carbono $\left(\mathrm{CO}_{2}\right)$. Além disso, também avaliar, através das frações do balanço de massa de carbono se o reservatório é fonte ou sumidouro de carbono, com ou sem produção primária e, eventualmente, se as fontes de carbono provém de fora do sistema aquático (alóctone) ou do próprio sistema (autóctone). Os resultados para o estudo de caso mostraram que o reservatório da PCH Salto Natal, para as condições de carga identificadas no monitoramento, é emissor de dióxido de carbono, com fontes de carbono que provém de fora do reservatório (alóctone) e também que não emite metano.

Palavras-chave: emissão de gases de efeito estufa, reservatório de PCH, balanço de carbono.

\section{INTRODUÇ̃̃O}

Na década de 1990, alguns estudos apresentaram evidências que reservatórios de usinas hidrelétricas (UHE) poderiam ser fontes potenciais (emissores potenciais) de gases de efeito estufa (GEE), (Rudd et al., 1993; Kelly et al., 1994), produto da decomposição da matéria orgânica, quer seja, alóctone, autóctone, ou ainda, inundada em sua bacia de acumulação durante a formação do reservatório. Neste caso, os gases produzidos são principalmente o dióxido de carbono $\left(\mathrm{CO}_{2}\right)$, o metano $\left(\mathrm{CH}_{4}\right)$ e o óxido nitroso $\left(\mathrm{N}_{2} \mathrm{O}\right)$, que comprovadamente interagem com a radiação infravermelha na atmosfera causando a intensificação do efeito estufa natural.

Este fato tornou-se um argumento significativo contra a construção de novas barragens de usinas hidrelétricas. Portanto, para justificar novos projetos de usinas, é importante mostrar, entre outros aspectos, que, em termos ambientais, esta é a melhor solução para uma dada capacidade instalada. Por conseguinte, o potencial de redução de gases de efeito estufa em relação a outras fontes de energia deve ser demonstrado.

Este artigo tem como objetivo apresentar um modelo matemático para avaliar a emissão de 
gases de efeito estufa de uma pequena central hidrelétrica $(\mathrm{PCH})$ utilizando desenvolvimento do balanço de carbono para o reservatório. Adicionalmente, pretende-se identificar as diferentes vias do ciclo do carbono, além de, avaliar por meio de um modelo de balanço de massa, em que condições de carga o reservatório comporta-se como uma fonte ou sumidouro de carbono e se esta fonte é alóctone ou autóctone.

Cabe destacar que a avaliação da origem da matéria orgânica, autóctone ou alóctone, é importante no estudo das emissões líquidas (foot print) do reservatório. Isto se explica porque os reservatórios podem processar matéria orgânica alóctone de algum lugar ao longo da bacia, independente da formação do reservatório.

No entanto, muitas pesquisas realizadas com este objetivo fundamentam seu procedimento metodológico para o monitoramento dos gases de efeito estufa sem considerar os aspectos do ciclo da matéria orgânica no reservatório e sua sinergia com as dinâmicas dos processos que compõe uma bacia hidrográfica (Fearnside, 2002; Hanson et al, 2004; Santos et al, 2005; e Sbrissia, 2008). Dentro deste contexto, este artigo adiciona uma abordagem que complementa os resultados existentes.

\section{ASPECTOS CONCEITUAIS}

$\mathrm{O}$ metano $\left(\mathrm{CH}_{4}\right)$ e o dióxido de carbono $\left(\mathrm{CO}_{2}\right)$ são os dois principais gases de efeito estufa associados a reservatórios de usinas hidrelétricas com forte impacto sobre o clima. Entretanto, o metano tem o potencial de aquecimento global 21 vezes maior que o dióxido de carbono (para um período de 100 anos) (Baird, 2001).

Os gases emitidos pelos reservatórios originam-se da decomposição de três fontes a saber: da biomassa original inundada; da biomassa formada pelo processo de fotossíntese nas águas do reservatório; e da matéria orgânica proveniente da bacia de drenagem. A decomposição diminui progressivamente a quantidade de biomassa inundada, e, portanto, a proporção da contribuição para a emissão de gases diminui com o tempo, sendo a maior contribuição gerada nos primeiros três anos após a inundação (Santos et al., 2005; Huttunem e Martikainem, 2005).

Quando as pesquisas sobre a importância das emissões dos gases de efeito estufa provenientes de reservatórios de usinas hidrelétricas começaram a apresentar resultados relevantes, o interesse era voltado principalmente ao destino da matéria orgânica biodegradável após a inundação da biomassa terrestre. Logo se tornou claro que a degradação da vegetação inundada era apenas uma parte do problema relacionado à emissão dos gases dos reservatórios. A degradação da matéria orgânica autóctone disponível não poderia explicar as altas emissões de metano e dióxido de carbono em determinados reservatórios. A quantidade de matéria orgânica de origem alóctone, proveniente das regiões a montante do reservatório, é responsável pela produção de uma parte significativa dos gases de efeito estufa formados no ambiente lêntico (Svensson, 2005).

A matéria orgânica pode ser decomposta tanto em ambientes aeróbios quanto anaeróbios. $\mathrm{Na}$ presença de oxigênio são produzidos dióxido de carbono e água, enquanto que na ausência de oxigênio metano e dióxido de carbono. Adicionalmente a estes, são formados (ácidos fúlvicos, ácidos húmicos e húmus). Estes compostos inertes são polímeros fenólicos originados principalmente da lignina, presente na madeira. Assim, parte do carbono originalmente presente é emitida na forma de gás, e a outra parte é transportada pela água como ácido fúlvico e húmico, solúveis na água. A parte insolúvel e a parte inerte dos resíduos fenólicos são incorporadas ao sedimento de fundo do reservatório (Santos et al., 2005). Sendo assim, a emissão dos gases de efeito estufa em reservatórios é controlada pelo transporte físico destes gases a partir do sedimento e da coluna de água. Os modos de transporte mais importantes em ambientes continentais são: a difusão molecular, processo ebulitivo e a advecção causada pela mistura turbulenta da água e transporte via plantas aquáticas (Huttunen e Martikainem, 2005).

A maioria dos estudos sobre emissões de gases de efeito estufa de reservatórios tem se concentrado na estimativa de gás metano. Águas lóticas e turbulentas são bem oxigenadas e não apresentam condições para a formação de metano, que é produto da degradação da matéria orgânica em condições anaeróbias. Em águas lênticas, particularmente em águas estratificadas, o hipolímnio é anóxico, e apresenta condições para a metanogênese. O metano, então formado, se difunde através da coluna de água ou forma bolhas que irão se mover em direção à superfície da água. Cabe destacar, que o metano que difunde é também um substrato para as bactérias metanotróficas e é rapidamente consumido por estes microorganismos, se houver disponibilidade de oxigênio. Portanto, se o corpo de água for profundo, somente uma pequena quantidade de metano alcançará a superfície da água. 
Quando a metanogênese é intensa, a concentração de oxigênio diminui, expandindo a zona anóxica. A zona oxigenada do reservatório irá diminuir e se a profundidade do corpo de água não for grande o suficiente, o processo de oxidação será insuficiente para remover todo o metano. Por outro lado, a ebulição, transporte de massa através das bolhas, faz o metano indisponível para as bactérias metanotróficas enquanto ele se move do sedimento do reservatório até a superfície da água, se difundindo diretamente na atmosfera (Svensson, 2005).

Ebulição e difusão parecem não ser os únicos fenômenos de emissão de gases de efeito estufa por reservatórios. Estudos recentes mostram que um das principais vias de emissão pode ser a difusão de gases quando a água passa na turbina da usina (Fearnside, 2002).

Finalmente, as emissões de reservatórios devem variar amplamente com a localização geográfica, tipo de vegetação do entorno do reservatório, temperatura, sazonalidade, tamanho e profundidade do reservatório, profundidade da tomada de água das turbinas, operação da barragem, dentre outros fatores (Fearnside 2002, Santos et al, 2005, Sbrissia, 2008). Todos estes elementos influenciam na dinâmica da matéria orgânica no reservatório que determina o respectivo padrão de emissão ao longo do tempo.

\section{MATERIAL E MÉTODOS}

Este item apresenta uma descrição da área de estudo, a definição da rede de amostragem, a freqüência de amostragem e os parâmetros analisados. Adicionalmente, apresenta a concepção do modelo matemático do ciclo do carbono no reservatório do sistema, baseada no ciclo do carbono, e também, a estratégia para a solução do sistema de equações de balanço de massa e para a estimativa dos parâmetros definidos no modelo.

\section{Caracterização da área de estudo}

Este estudo foi desenvolvido no reservatório da PCH Salto Natal, que possui potência instalada de 15 MW e de propriedade da BRASCAN Energética S/A, subsidiária brasileira da Brookfield Renewable Power. Está localizada no rio Mourão, Figura 1, afluente da margem esquerda do rio Ivaí, na bacia do Paraná, situando-se no paralelo $24^{\circ} 04^{\prime} 20^{\prime} \mathrm{S}$ e meridiano $52^{\circ} 17^{\prime} 30^{\prime \prime} \mathrm{W}$ a, aproximadamente, $8 \mathrm{~km}$ da área urbana do município de Campo Mourão, a jusante da UHE Mourão I, de propriedade da Companhia Paranaense de Energia - COPEL e, aproximadamente, $95 \mathrm{~km}$ da foz do rio Mourão (COPEL, 2000).

A área de drenagem do reservatório é de $585 \mathrm{~km}^{2}$, e o nível de água esta na cota $510 \mathrm{~m}$. O reservatório possui uma área de 62 ha, sendo que 17 ha correspondem ao leito do rio e um volume de $4,25 \mathrm{hm}^{3}$, o que corresponde a uma vazão média de longo termo de $17,0 \mathrm{~m}^{3} / \mathrm{s}$, e com um tempo de residência de aproximadamente três dias (COPEL, 2000). A profundidade média do reservatório é de $15 \mathrm{~m}$.

O clima na região da PCH Salto Natal, segundo a classificação de Köeppen é Cfa, que indica "clima subtropical com temperatura média no mês mais frio inferior a $18^{\circ} \mathrm{C}$ (mesotérmico) e temperatura média no mês mais quente acima de $22^{\circ} \mathrm{C}$, com verões quentes, geadas pouco freqüentes e tendência de concentração das chuvas nos meses de verão, contudo sem estação seca definida" (COPEL, 2000).

A precipitação média anual na região da PCH Salto Natal é de aproximadamente 1.643 $\mathrm{mm} / \mathrm{ano}$; a máxima mensal quando observada foi de $428 \mathrm{~mm}$ em dezembro de 1964; o mês que apresentou maior média foi dezembro $(222,2 \mathrm{~mm})$; a precipitação média anual resultou em 1.679 $\mathrm{mm} / \mathrm{ano}$; a direção predominante do vento é leste (E) em todos os meses (COPEL, 2000).

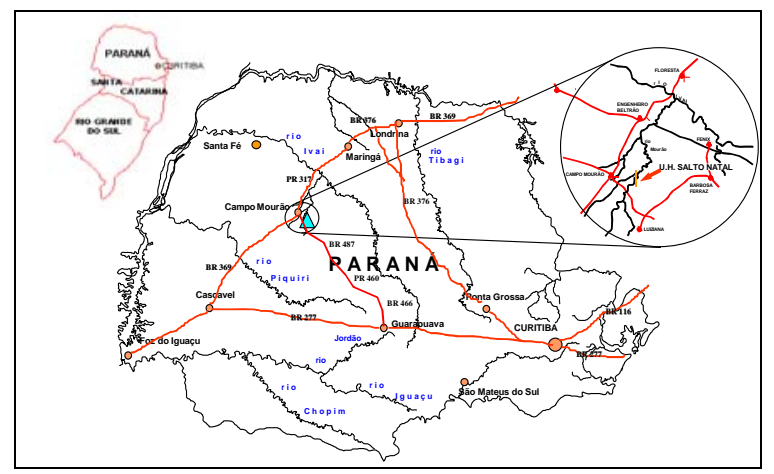

Figura 1 - Localização da PCH Salto Natal

A tipologia vegetal da área do projeto pode ser considerada como adotando o sistema de classificação da COPEL (2000), incluindo: Sistema Primário de Floresta Estacional Semidecidual Montana - FE e de Floresta Estacional Semidecidual Aluvial FA; Sistema Secundário de $4^{\mathrm{a}}$ Fase da Sucessão Vegetal - C4 (capoeira) e $5^{\text {a }}$ Fase da Sucessão Vegetal - C5 
(capoeirão/floresta secundária); Agropecuária - AP; Reflorestamento - RE; Sistema Primário incluindo: Floresta Estacional Semidecidual Montana - FE.

Quanto à área inundada, aproximadamente $50 \%$ era formada por pastagens, $30 \%$ por cultura de soja e $20 \%$ por mata ciliar (COPEL, 2000), indicando uma característica típica da região onde está inserido o reservatório.

\section{Rede de amostragem e freqüência de coleta das amostras}

Para a coleta das amostras foram selecionados locais levando-se em consideração aspectos relacionados a acesso, hidrodinâmica do reservatório e profundidade (Sbrissia, 2008): O Ponto 1 (2403'29', S, 52 $\left.{ }^{\circ} 17^{\prime} 22^{\prime \prime N}\right)$ localizado a jusante do reservatório, na saída das turbinas, foi escolhido para fornecer elementos para estudo de balanço de carbono e qualidade da água. O Ponto 2 $\left(24^{\circ} 05^{\prime} 22^{\prime}\right.$ 'S, $\left.52^{\circ} 18^{\prime} 05^{\prime \prime} \mathrm{N}\right)$ : localizado no reservatório, próximo a barragem, definido para fundamentar os estudos de balanço de carbono e estratificação térmica e química do reservatório; O Ponto 3

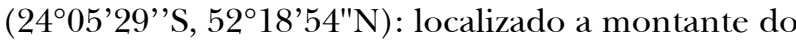
reservatório da PCH Salto Natal, no rio Mourão, principal tributário do reservatório, representa o ponto de controle de qualidade da água na entrada do reservatório. Neste estudo, foram realizadas quatro campanhas, no período de maio de 2007 a março de 2008, limitadas pelas condições de financiamento existentes à época. Os detalhes e dados obtidos encontram-se detalhados em Sbrissia (2008).

\section{Obtenção dos dados limnológicos da superfície e coluna de água}

As variáveis analisadas foram: alcalinidade total, condutividade, demanda bioquímica de oxigênio (DBO), demanda química de oxigênio (DQO), oxigênio dissolvido (OD), pH, temperatura do ar, temperatura da água, sólidos totais (ST), sólidos suspensos totais (SST), sólidos dissolvidos totais (SDT), sólidos sedimentáveis (SS), turbidez, nitrogênio orgânico, nitrogênio amoniacal, nitrito, nitrato, fósforo total, carbono orgânico total (COT), carbono orgânico total dissolvido (COD), carbono inorgânico dissolvido (CID), carbono orgânico particulado (COP) e profundidade com disco de Secchi em acordo com banco de dados apresentado integralmente em Sbrissia (2008). Todos os ensaios foram realizados integralmente no Laboratório de Engenharia Ambiental Francisco Borsari Netto (Labeam) e em acordo com APHA (1998).
O carbono orgânico total (COT), o carbono orgânico dissolvido (COD), carbono inorgânico dissolvido (CID) e o carbono orgânico particulado (COP) foram determinados utilizando o método de combustão à alta temperatura (Shimadzu, 2006), em equipamento TOC-VCPH, marca Shimadzu. As amostras para a determinação do COD foram filtradas em membranas de ésteres de celulose com porosidade de 0,45 $\mu \mathrm{m}$, marca Millipore. O COP foi estimado pela diferença entre o COT e o COD. O teor de carbono orgânico no sedimento (CS) foi determinado utilizando-se do equipamento LECO modelo C-144.

\section{Balanço de massa do carbono}

Compreender o ciclo de carbono em um reservatório é fundamental para avaliar se este corpo hídrio é uma fonte ou um sumidouro de gases de efeito estufa. Entender como um lago processa o carbono requer quantificar as cargas de carbono alóctone e autóctone, conhecer os processos biológicos e físico-químicos e, ainda, compreender os efeitos combinados das cargas de carbono no metabolismo do lago, no processo de sedimentação e no fluxo para atmosfera (Hanson et al., 2004).

O uso de um modelo matemático pode auxiliar na interpretação de questões que seriam difíceis de responder por medidas diretas como, por exemplo, qual é o destino do carbono alóctone no lago; como as cargas de carbono exógeno alteram o ciclo de carbono no lago; e a partir de qual carga de carbono um lago se torna um emissor de gases de efeito estufa para atmosfera (Hanson et al., 2004).

Para representar este sistema foi definido um modelo determinístico, em estado não estacionário e contínuo para ser aplicado a um pequeno reservatório (Tucci, 2002). Este modelo considera o ciclo de carbono interno do reservatório, e também as trocas de fluxo de carbono com os sistemas terrestres e atmosféricos. Modelos determinísticos representam os fenômenos físicos de um determinado sistema e as equações matemáticas resultantes são derivadas do transporte de massa e fenômenos de reação (Braga, 2001).

O reservatório foi considerado um reator homogêneo bem misturado, portanto, a modelagem realizada neste estudo é válida somente para os períodos em que o reservatório não está estratificado. Não foi considerada a morfometria do reservatório e, tão pouco, a separação dos ambientes litorâneos e pelágicos. Para simplificar o sistema, o reservatório foi considerado um cilindro com profundidade definida como a profundidade média do reservató- 
rio. No balanço de massa, não foi incluído o sistema de carbonato de cálcio $\left(\mathrm{CaCO}_{3}\right)$.

Neste modelo, as trocas de carbono orgânico e inorgânico entre o sedimento e a coluna de água, foram consideradas de ocorrência na camada superficial do sedimento. A Figura 2 mostra um diagrama simplificado de um lago idealizado situado numa bacia hidrográfica, onde estes processos estão representados em seus respectivos compartimentos.

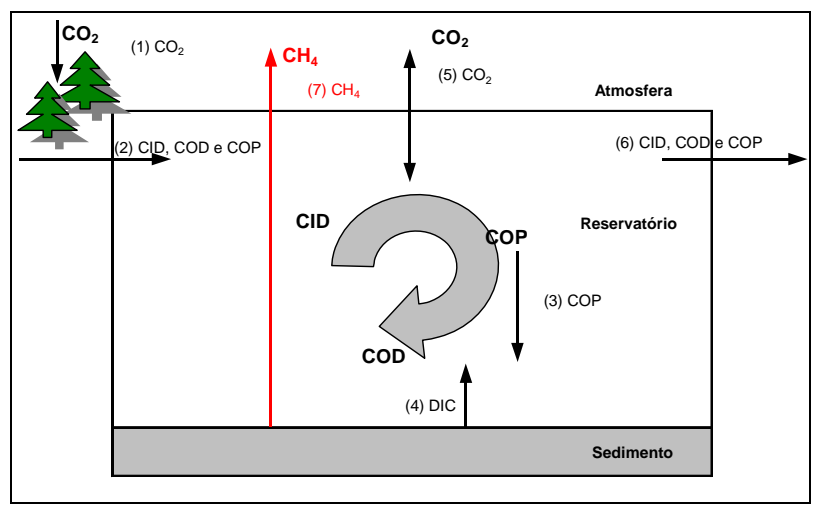

Figura 2 - Diagrama simplificado do ciclo de carbono em um reservatório

O carbono foi agrupado em quatro compartimentos, a saber: carbono inorgânico dissolvido (CID), carbono orgânico dissolvido (COD) e carbono orgânico particulado (COP) e carbono orgânico no sedimento (CS). O ciclo do carbono descrito de maneira simplificada ocorre da seguinte maneira: (1) A produção primária líquida (PPL) em sistemas terrestres resulta na acumulação de biomassa; (2) A parte da biomassa chega ao lago através da água superficial como COD, CID e COP. O carbono no lago apresenta formas orgânica e inorgânica. Produtores primários transformam CID em COP e excretam COD neste processo. A decomposição do COP está associada a produção de CID e COD. A respiração microbiana e a fotodegradação mineraliza o COD na forma de CID; (3) Uma parte do COP sedimenta e, da parte restante, uma fração é composta por microorganismos fotossintetizantes, $\mathrm{COP}_{\mathrm{v}}$, cujo metabolismo produz COD e CID. Parte do Carbono Orgânico Particulado pode ter seu ciclo de vida representado por uma taxa de mortalidade $\left(\mathrm{COP}_{\mathrm{m}}\right)$ produzindo potenciais alterações no balanço de Carbono; (4) o COP no sedimento mineraliza lentamente e libera CID para a coluna de água no processo; (5) o gradiente da pressão parcial do $\mathrm{CO}_{2}$ entre o lago e a atmosfera determina o fluxo atmosférico líquido (FAL) do CID; (6) todas as formas de carbono são transportadas para fora do lago através da água superficial; (7) o COD gerado por diagênese (transformação do carbono particulado em carbono dissolvido) no sedimento é transformado por bactérias em metano. Por ser relativamente insolúvel na água, o gás metano produzido no sedimento é transportado diretamente para atmosfera na forma de bolhas;

A Tabela 1, a seguir, mostra as taxas de reação entre os compartimentos do modelo (coluna 1), apresentados na figura 2, e enfatizam os principais processo da dinâmica da Matéria orgânica (coluna 2). A Figura 3 mostra o diagrama conceitual do modelo de balanço de massa proposto.

O sistema em estudo pode ser representado pelas seguintes equações de balanço de massa de acordo com os compartimentos de CID, COD, COP e CS:

a)Balanço de massa do carbono inorgânico dissolvido $\left(C_{A}=C I D\right)$

$V \frac{d C_{A}}{d t}=Q C_{A i n}-Q C_{A}-k_{A C} C_{A} V+k_{B A} C_{B} V$

$+k_{C A} C_{C} V+k_{D A} C_{D} V+$

$k_{E A} C_{E} V S-K_{\text {Adif }} C_{A}$

b) Balanço de massa do carbono orgânico dissolvido $\left(C_{B}=C O D\right)$

$V \frac{d C_{B}}{d t}=Q C_{B i n}-Q C_{B}-k_{B A} C_{B} V+k_{C B} C_{C} V$

Tabela 1 - Taxas de reação entre os compartimentos do modelo

\begin{tabular}{c|l|c}
\hline $\begin{array}{c}\text { Parâmetro } \\
\mathbf{( 1 )}\end{array}$ & \multicolumn{1}{|c|}{$\begin{array}{c}\text { Processo } \\
\mathbf{( 2 )}\end{array}$} & $\begin{array}{c}\text { Valor (dia } \\
\mathbf{( 3 )}\end{array}$ \\
\hline$k_{a c}$ & Fotossíntese & $-2,195910^{-10}$ \\
\hline$k_{b a}$ & Respiração Aeróbia & $+6,871310^{-04}$ \\
\hline$k_{c a}$ & Respiração Aeróbia & $+2,744310^{+05}$ \\
\hline$k_{c b}$ & Excreção & $-1,782910^{+05}$ \\
\hline$k_{c d}$ & Mortalidade & $+6,327410^{-01}$ \\
\hline$k_{c e}$ & Sedimentação & $-9,613210^{+04}$ \\
\hline$k_{d a}$ & Respiração Aeróbia & $+5,052010^{+05}$ \\
\hline$k_{d e}$ & Sedimentação & $-5,052010^{+05}$ \\
\hline$k_{e a}$ & Respiração Aeróbia & $+6,145010^{-04}$ \\
\hline$k_{e c}$ & Ressuspensão & $-3,011010^{-13}$ \\
\hline$k_{e d}$ & Ressuspensão & $-4,325910^{-15}$ \\
\hline$k_{e e b}$ & Fluxo Ebulitivo & $-6,252010^{-04}$ \\
\hline$k_{a d i f}$ & Fluxo Difusivo & $+4,837010^{-01}$ \\
\hline & &
\end{tabular}




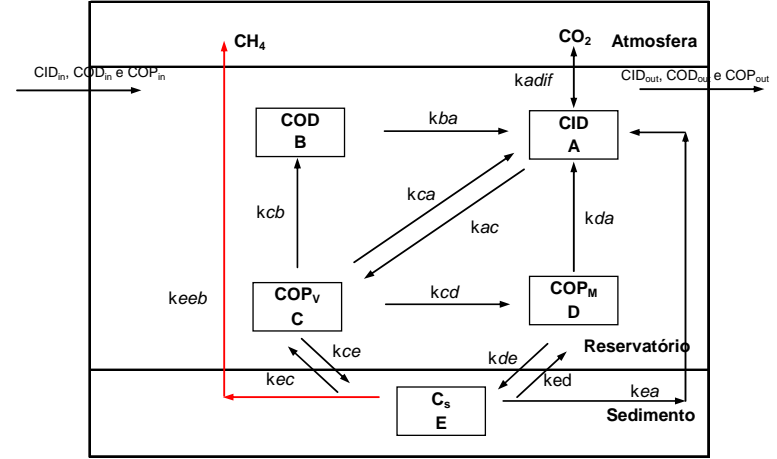

Figura 3 - Diagrama do modelo.

c) Balanço de massa do carbono orgânico particulado vivo $\left(C_{C}=C O P V\right)$

$$
\begin{aligned}
& V \frac{d C_{C}}{d t}=Q C_{C i n}-Q C_{C}-k_{C A} C_{c} V-k_{C B} C_{C} V-k_{C D} C_{C} V \\
& -k_{C E} C_{C} V+k_{A C} C_{A} V+k_{E C} C_{E} V
\end{aligned}
$$

d)Balanço de massa do carbono orgânico particulado morto $\left(C_{D}=C O P M\right)$

$$
\begin{aligned}
& V \frac{d C_{D}}{d t}=Q C_{D i n}-Q C_{D}-k_{D A} C_{D} V-k_{D E} C_{D} V \\
& +k_{C D} C_{C} V+k_{E D} C_{E} V
\end{aligned}
$$

e) Balanço de massa do carbono orgânico total no sedimento $\left(C_{E}=C S\right)$

$$
\begin{aligned}
& V S \frac{d C_{E}}{d t}=Q C_{E i n}-Q C_{E}+k_{C E} C_{c} V+k_{D E} C_{D} V-k_{E C} C_{E} V \\
& -k_{E D} C_{E} V S-k_{E A} C_{E} V S-k_{E e b} C_{E} V S
\end{aligned}
$$

em que: $\mathrm{V}$ é volume do lago $\left[\mathrm{L}^{3}\right]$; VS é volume do sedimento $\left[\mathrm{L}^{3}\right] ; \mathrm{C}_{i}$ é a concentração do componente $\left[\mathrm{ML}^{-3}\right]$; t é o tempo [T]; Q é a vazão volumétrica $\left[\mathrm{L}^{3} \mathrm{~T}^{-1}\right] ; \mathrm{C}_{\text {in }}$ é a concentração influente do componente $i\left[\mathrm{ML}^{-3}\right] ; \mathrm{k}_{m n}$ é a taxa de reação do componente $m$ em $n\left[\mathrm{~T}^{-1}\right] ; \mathrm{k}_{n m}$ é a taxa de reação do componente $n$ em $m\left[\mathrm{~T}^{-1}\right] ; \mathrm{k}_{k j}$ é a taxa de sedimentação do componente $k,\left[\mathrm{~T}^{-1}\right] ; \mathrm{k}_{j k}$ é a taxa de ressuspensão do componente $j\left[\mathrm{~T}^{-1}\right]$.

\section{Estimativa dos Parâmetros e \\ Estratégias de Simulação}

Os parâmetros do modelo, como apresentado na eqs. 1 a 5 , foram estimados por meio de um conjunto de ferramentas de identificação de sistemas (System Identification Toolbox) do programa computacional MATLAB 7, mais especialmente rotinas computacionais com base no Método de Minimização por Predição de Erro (PEM) (The Mathworks Inc., 2004). Esta técnica tem sido utilizada para a solução de sistemas dinâmicos (regimes não estacionários ou não permanentes) em diversas áreas do conhecimento como processamento de sinais, modelagem de processos biológicos e bioquímicos, processos estocásticos dentre outros (Dennis e Schnabel, 1983; Sung e Lee, 2001; Farina e Piroddi , 2010).

A idéia básica deste método de estimativa de parâmetros em sistemas dinâmicos, como o aqui proposto, é o de construir um algoritmo preditor iterativo e comparar suas predições com dados disponíveis (dados de monitoramento) usando uma medida de otimização para o erro, por exemplo, minimizar o erro quadrático da predição, como o utilizado nesta pesquisa. As iterações são satisfeitas quando ou o número máximo de iterações é alcançado, ou o gradiente de erro é menor que a tolerância, ou quando um valor melhor do objetivo não pode ser encontrado (Dennis and Schabel , 1983; Qingchang, 1990; Soderstrom et al, 1991; Ljung, 1999; Sung e Lee, 2001; Ljung, 2002; Borjas e Garcia, 2004; Li e Zhang, 2006; Farina e Piroddi, 2010).

Para tanto, duas rotinas computacionais foram desenvolvidas em ambiente MATLAB 7 (The Mathworks Inc., 2004) e apresentadas em Sbrissa (2008). A primeira contempla a declaração das matrizes das equações de estado (eqs 1 a 5). A $2^{\underline{a}}$ rotina, por sua vez, é lida pela rotina principal do modelo onde as matrizes do sistema de equações e os parâmetros são estimados com base em algoritimo proposto em Dennis e Schnabel (1983), e representado pela subrotina 'pem' (The Mathworks Inc., 2004).

\section{Produção líquida do ecossistema (PLE) e fluxo de carbono líquido (FCL)}

As frações do balanço de massa do carbono podem fornecer medidas convenientes para classificar o nível trófico e os fluxos de emissões em lagos. A produção líquida do ecossistema (PLE) é a diferença entre a fração de massa da produção primária bruta (PPB), fotossíntese, e as frações de massa da respiração $(\mathrm{R})$ :

$$
P L E=P P B-R
$$

Esta equação descreve o balanço metabólico do sistema aquático. A PLE negativa significa que o 
sistema é alóctone, fontes externas de carbono alimentam a respiração $(\mathrm{R})$ além da $\mathrm{PPB}$, enquanto a PLE positiva um sistema autóctone.

O fluxo de carbono líquido (FCL) representa o balanço entre fluxo atmosférico líquido (FAL) e o sedimento $(\mathrm{S})$ :

$$
F C L=F A L-S
$$

Esta equação descreve o lago como uma fonte de carbono para a atmosfera (FCL positivo) ou como um sumidouro de carbono (FCL negativo).

\section{RESULTADOS E DISCUSSÕES}

\section{Resultados do monitoramento da qualidade da água}

Os resultados das análises do monitoramento físico, químico e biológico, apresentados em Sbrissia (2008), mostram que as variáveis analisadas atendem ao limite da Classe 2 da Resolução CONAMA 357/2005. Os resultados encontrados indicam que a qualidade da água do reservatório da PCH Salto Natal é fortemente influenciada pela presença do reservatório da UHE Mourão I, a montante, em especial pelo aporte pouco significativo de transporte de sólidos e baixa dinâmica de matéria orgânica (Sbrissia, 2008).

A maior parte dos sedimentos da bacia de drenagem do reservatório da PCH Salto Natal fica retida no reservatório da UHE Mourão I. Como consequência, as concentrações das variáveis analisadas são bastante baixas, principalmente os nutrientes, o que faz com que o reservatório seja predominantemente oligotrófico. O reservatório, em conseqüência da escassez de nutrientes, apresenta baixa produtividade.

Este fato afeta o ciclo do carbono no reservatório e, portanto, as emissões de dióxido de carbono. As frações de dióxido de carbono características do reservatório são resultado do aporte de matéria orgânica na sua entrada e da decomposição da matéria orgânica do sedimento, não podendo ser transformadas em carbono orgânico particulado vivo $\left(\mathrm{COP}_{\mathrm{V}}\right)$. Restando apenas, para o dióxido de carbono, sua saída através da vazão efluente e da emissão para a atmosfera (Sbrissia, 2008).

\section{Resultados da modelagem}

Os resultados da solução do sistema de equações (eq. 1 a eq. 5) e da identificação para os valores das taxas de reação para as espécies de carbono do balanço de massa, podem ser observados na Figura 4. Os resultados para esta simulação, indicam que o erro de estimativa entre os valores medidos e simulados para as variáveis carbono inorgânico dissolvido (CID), carbono orgânico dissolvido (COD) e para o carbono orgânico total no sedimento (CS) foi respectivamente $-3,2 \%,-1,3 \%$ e $-0,4 \%$.

Os resultados desta análise permitiram definir os valores dos parâmetros do sistema identificados pelo modelo (Tabela 2). A Figura 5 mostra o diagrama conceitual do modelo com os valores dos parâmetros.

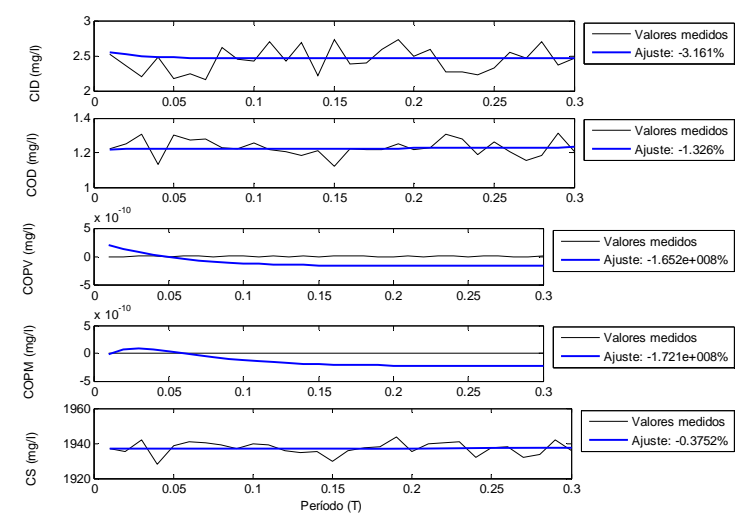

Figura 4 - Resultado da simulação para os dados de monitoramento

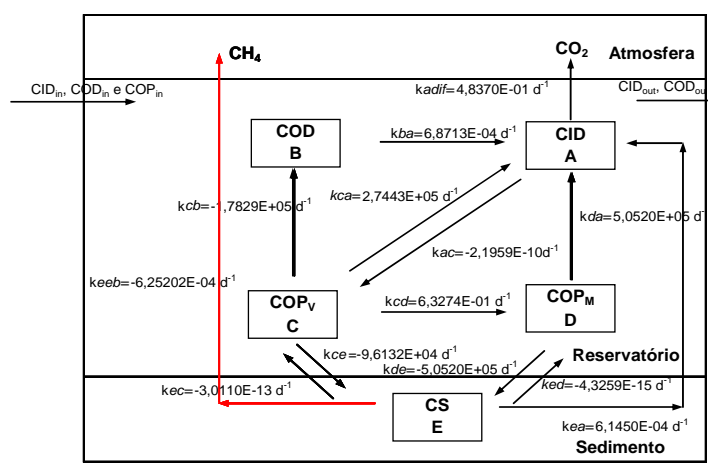

Figura 5 - Taxas de reação obtidas pelo modelo

Os resultados parciais do balanço de massa $\left(-k_{\text {adif }}\right.$ CID e $-k_{\text {eeb }}$ CS) para três dias, são apresentados na coluna 3 da Tabela 1, a partir dos quais foram calculados os fluxos difusivos e ebulitivos em $\mathrm{kg} \mathrm{d}^{-1}$ $\mathrm{km}^{-2}$. A Tabela 3 mostra também os resultados dos cálculos do fluxo de carbono líquido (FCL) e produção líquida do ecossistema (PLE). Conforme 
descrito na metodologia, o FCL, que é a diferença entre as frações de balanço de massa do fluxo atmosférico líquido de carbono (FAL) e as frações que sedimentam (S), indica se o reservatório, nas condições de carga do balanço de massa, é uma fonte (FCL positivo) ou sumidouro (FCL negativo) de carbono. A PLE, que representa a diferença entre as frações de balanço de massa que são absorvidas pelo processo de fotossíntese e as frações de balanço da respiração, indica se o reservatório é autóctone (com produção primária) (PLE positiva) ou alóctone (PLE negativa).

Tabela 3 - Resultado do cálculo dos fluxos de emissão, fcl e ple.

\begin{tabular}{|c|c|c|c|c|c|c|}
\hline Dia & $\begin{array}{l}\text {-kadifCID } \\
\left(\mathrm{mg} \mathrm{l}^{-1} \mathrm{~d}^{-1}\right)\end{array}$ & $\begin{array}{c}\text { Fluxo difusivo } \\
\left(\mathrm{kg} \mathrm{d}^{-1} \mathrm{~km}^{-2}\right)\end{array}$ & $\begin{array}{l}\text {-keebCS } \\
\left(\mathrm{mg} \mathrm{l}^{-1} \mathrm{~d}^{-1}\right)\end{array}$ & $\begin{array}{l}\text { Fluxo ebulitivc } \\
\left(\mathrm{kg} \mathrm{d}^{-1} \mathrm{~km}^{-2}\right)\end{array}$ & $\begin{array}{c}\mathrm{FCL}=\mathrm{FAL}-\mathrm{S} \\
\left(\mathrm{mg} \mathrm{I}^{-1}\right)\end{array}$ & $\begin{array}{c}\text { PLE=PPB-R } \\
\left(\left.m g\right|^{-1}\right)\end{array}$ \\
\hline 1 & $-1,224$ & $-8388,684$ & 1,211 & 121,095 & 0,013 & $-1,192$ \\
\hline 2 & $-1,212$ & $-8305,792$ & 1,211 & 121,095 & 0,001 & $-1,192$ \\
\hline 3 & $-1,204$ & $-8251,746$ & 1,211 & 121,095 & $-0,007$ & $-1,192$ \\
\hline Média & $-1,2131$ & $-8315,4076$ & 1,2109 & 121,0950 & 0,0021 & $-1,1920$ \\
\hline
\end{tabular}

Os resultados dos cálculos mostram que o fluxo difusivo médio para atmosfera, para os três primeiros dias, foi de $-8.315 \pm 8,0 \% \mathrm{~kg} \mathrm{~d}^{-1} \mathrm{~km}^{-2}$. Quanto ao fluxo ebulitivo, a média para os três primeiros dias foi de $121 \pm 5,5 \mathrm{~kg} \mathrm{~d}^{-1} \mathrm{~km}^{-2}$. O sinal positivo do fluxo ebulitivo indica que o reservatório não está emitindo metano e, portanto, o fluxo de gases do compartimento do sedimento para atmosfera por ebulição é nulo. O erro foi calculado somando-se o ajuste do modelo entre os dados de campo e simulado mais o erro do método analítico do carbono. A análise do valor médio do FCL mostra que o reservatório, para o tempo de residência de três dias é uma fonte de emissão de carbono. A média da PLE indica que o reservatório é alóctone (heterotrófico), isto é, as fontes de carbonos do reservatório são externas.

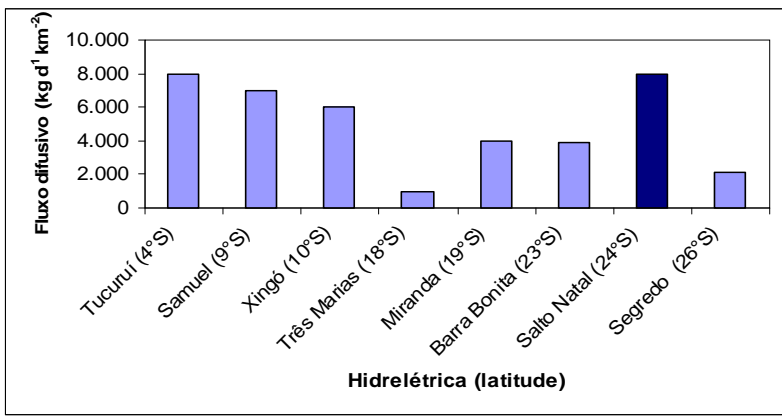

Figura 5 - Comparação entre os dados do inventário brasileiro de emissões de $\mathrm{CO}_{2}$ e os estimados pelo modelo para PCH Salto Natal

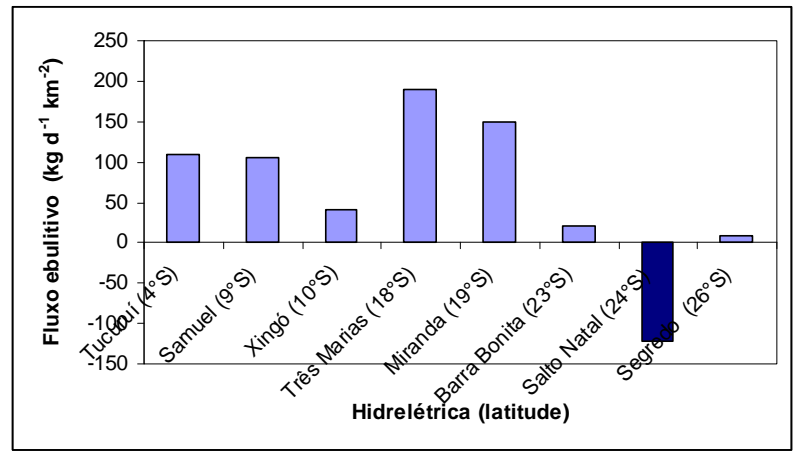

Figura 6 - Comparação entre os dados do inventário brasileiro de emissões de $\mathrm{CH}_{4}$ e os estimados pelo modelo para PCH Salto Natal.

Nas Figuras 5 e 6 são apresentados os resultados obtidos pelo modelo para a PCH Salto Natal e comparados com os dados do inventário brasileiro de emissões (MCT, 2006), respectivamente para fluxos difusivo $\left(\mathrm{CO}_{2}\right)$ e ebulitivo $\left(\mathrm{CH}_{4}\right)$.

Os resultados produzidos pelo modelo para o fluxo difusivo, Figura 5, mostram que esta emissão é expressiva em relação aos reservatórios das outras usinas hidrelétricas. A princípio, em função da localização geográfica, era esperado que os resultados das emissões difusivas para o estudo de caso fossem mais próximos dos resultados das usinas hidrelétricas situadas em latitudes semelhantes. Entretanto, na avaliação do fluxo ebulitivo $\left(\mathrm{CO}_{2}\right)$, Figura 6 , o valor negativo indica a ausência de fluxo de gases do compartimento do sedimento para a atmosfera.

Alguns fatores devem ser considerados na análise destes resultados. A metodologia utilizada para a avaliação dos fluxos pelo inventário MCT (2006) é diferente daquela utilizada por este estudo, o que pode ter contribuído para as diferenças dos fluxos entre os dois métodos de avaliação. Assim, para avaliar estas diferenças seria necessário calcular as emissões nestes reservatórios utilizando este modelo.

Outro fator que deve ser considerado é que os resultados dos cálculos das frações parciais do balanço de massa para cada processo mostram um fluxo significativo do compartimento do carbono orgânico total do sedimento (CS) para o compartimento do carbono inorgânico dissolvido (CID) na coluna de água, o que significa que parte significativa do fluxo difusivo se deve a processos aeróbios no sedimento.

As condições de amostragem, tanto temporal como espacial da água e do sedimento, a espessura da camada ativa do sedimento, a maneira como 
foram estimados os valores médios do CS e a quantidade de carbono orgânico refratário no sedimento são alguns fatores que podem também ter influenciado na diferença entre os resultados entre os dois métodos de avaliação.

Quanto ao impacto da inundação da área para a formação do reservatório sobre a emissão de gases de efeito estufa é importante ressaltar que as concentrações de carbono orgânico no sedimento no trecho final do reservatório são significativamente maiores que nas outras áreas, conforme apresentado em Sbrissia (2008), trecho intermediário e próximo à barragem. Este fato é um indicativo que a origem do fluxo difusivo não é a matéria orgânica inundada durante a formação do reservatório. Por conseguintes, pode-se considerar que a matéria orgânica da qual o fluxo difusivo se origina é alóctone.

Devido ao fato que não foram detectados carbono orgânico particulado vivo $\left(\mathrm{COP}_{\mathrm{V}}\right)$ e carbono orgânico particulado morto $\left(\mathrm{COP}_{\mathrm{M}}\right)$ no monitoramento (Sbrissia, 2008), é provável que a origem da alta concentração de carbono no sedimento sejam cargas instantâneas de carbono particulado, que não foram identificadas durante o monitoramento. Estas cargas instantâneas sedimentam no trecho final do reservatório, quando a água muda de velocidade e passa de ambiente lótico para lêntico. Uma possibilidade de carga instantânea são as descargas de fundo realizadas pela UHE Mourão I, localizada a montante do reservatório da PCH Salto Natal. Mas para uma avaliação mais precisa deste processo são necessários estudos de uso de solo na bacia de influência do reservatório e de avaliação hidrológica e hidrodinâmica do reservatório.

\section{CONCLUSÕES}

O modelo de balanço de carbono é uma ferramenta fundamental não só para avaliar o ciclo biogeoquímico do carbono, como também para avaliar o potencial de emissão de gases de efeito estufa do reservatório.

Através da medição do carbono na coluna de água foi possível avaliar, se o reservatório é uma fonte ou sumidouro de carbono, isto é se é autóctone ou alóctone, do ponto de vista da produção primária dentro do reservatório. O método de predição de erro se mostrou uma técnica adequada para a solução do sistema de equações como um procedimento de calibração entre as concentrações de carbono medidas no monitoramento e as simuladas pelo modelo.

Quanto à comparação entre os valores de emissão do dióxido de carbono estimados pelo modelo e os valores medidos no inventário brasileiro de emissão (MCT, 2006), vale ressaltar que a metodologia desenvolvida neste estudo não substitui a metodologia do MCT (2006), entretanto, pode ser utilizada como um instrumento para compreender o processo de emissão de gases do efeito estufa em reservatórios.

O reservatório da PCH Salto Natal, de acordo com os dados de monitoramento e com os resultados da modelagem, apresenta dois caminhos para a diferença entre as concentrações de carbono inorgânico dissolvido (CID). Mais especificamente, esta diferença representa sua absorção por organismos fotossintetizantes ou a emissão para fora do reservatório. Como não foram detectados organismos fotossintetizantes, as concentrações de carbono orgânico particulado vivo $\left(\mathrm{COP}_{\mathrm{V}}\right)$ encontradas foram praticamente nulas, por conseguinte, o único caminho possível para o dióxido de carbono remanescente é a emissão para a atmosfera (Sbrissia, 2008).

A análise do fluxo atmosférico líquido (FAL) mostra que o reservatório apresenta uma propensão a ser fonte de carbono para a atmosfera, devido às baixas concentrações de $\mathrm{COP}_{\mathrm{M}}$ e $\mathrm{COP}_{\mathrm{V}}$, resultando em uma taxa de sedimentação muito baixa no reservatório.

A análise do balanço de massa permite concluir que o reservatório é fonte de emissão de dióxido de carbono, sendo que a matéria orgânica que causa a emissão é alóctone. Esta implicação é importante para a avaliação do impacto da formação do reservatório. Evidencia-se que a matéria orgânica sofreria decomposição na coluna de água do rio, mesmo sem a formação do reservatório, devido à sedimentação da matéria orgânica em trechos do rio onde a velocidade da água fosse mais baixa.

Durante as campanhas de verão, observaram-se eventos de emissão por bolhas, fluxo ebulitivo, no ponto $\mathrm{P} 3$, trecho do rio a montante do reservatório. Este ponto é caracterizado por indicar a diminuição da velocidade da água (mudança de ambiente lótico para lêntico), indicando a sedimentação da matéria orgânica proveniente da carga instantânea cuja origem pode ser a descarga de fundo da barragem da UHE Mourão I. Se não houvesse reservatório, esta carga provavelmente iria sedimentar ao longo do rio e causar emissões mais distribuídas, mas de mesmo impacto no balanço global da bacia hidrográfica. É importante ressaltar que para conclusões mais precisas são necessários mais estu- 
dos, considerando toda a bacia hidrográfica. Finalmente, a análise das simulações do modelo para o fluxo ebulitivo mostra que para os resultados do monitoramento e condições de carga simuladas não há fluxo do sedimento para a atmosfera, portanto sem emissão de metano para a atmosfera.

\section{AGRADECIMENTOS}

À Brascan Energética, por permitir o estudo do reservatório da PCH Salto Natal e ao CNPq/CTHidro, pela bolsa concedida para a realização desta pesquisa. Aos professores Sérgio Michelotto Braga (nosso anjo da guarda) e aos companheiros de viagem Heloise Garcia Knapik, Carolina Fagundes Caron e Luiz Guilherme Quintas Rossigneux, sem a ajuda dos quais este trabalho não seria realizado.

\section{REFERÊNCIAS}

APHA. (1998). "Standard Methods for the Examination of Water and Waste Water". 20. ed. Washington.

BAIRD, C. (2001). "Environmental Chemistry". Second Edition". New York: W. H. Freeman Company, 2001.

BORJAS, S. D. e GARCIA, C. (2004) "Modelagem de FCC usando Métodos de Identificação por Predição de Erro e por Sub-Espaços". IEEE Latin America Transactions, vol. 2(2), 108-113.

BRAGA, M. C. B. (2001). "Surface Water Quality Modelling of Mercury Contaminations". Thesis (Doctor of Philosophy). T.H. Huxley School of Environment, Earth Sciences and Engineering, University of London, 2001.

CONAMA - Conselho Nacional do Meio Ambiente. (2005).Resolução n. 357, de 17 de março de 2005. "Dispõe sobre a classificação e diretrizes ambientais para o enquadramento dos corpos de água superficiais, bem como estabelece as condições e padrões de lançamento de efluentes". Diário Oficial da União, Brasília, 18 de março de 2005.

COPEL - Companhia Paranaense de Energia.(2000). "Plano de Controle Ambiental da PCH de Salto Natal".

DENIS, J. e SCHNABEL, R. "Numerical Methods for Unconstrained Optimization and Nonlinear Equations" (1983). Prentice Hall, Inc, Englewood Cliffs, New Jersey.

FARINA, M. e PIRODDI, L. (2010). "Simulation error minimization identification based on multi-stage prediction". International Journal of Adptive Control and Signal
Processing. Published online in Wiley Online Library. DOI - 10.1002/acs.1203.

FEARNSIDE, P.M. (2002). "Greenhouse Gas Emissions from a Hydroelectric Reservoir (Brazil's Tucuruí dam) and Energy Policy Implication". Water and Soil Pollution.

HANSON P. C.; POLLARD A. I.; BADE D.L.; FREDICK K.; CARPENTER S. R.; FOLEY J.A. (2004). "A model of carbon evasion and sedimentation in temperate lakes". Global Change Biology, v. 10, n. 8, p. 12851298.

HUTTUNEM, J. T.; MARTIKAINEN P. J. (2005). "Long-term net methane release from finnish hydro reservoirs". Seminar on Greenhouse Fluxes from Hydro Reservoir and Workshop on Modeling Greenhouse Gas Emissions from Reservoir at Watershed Level". Rio de Janeiro. ELETROBRAS, COPPE/UFRJ.

KELLY, C.A. et al. (1994). "Turning Attention to Reservoirs Surfaces, a Neglected Area in Greenhouse Studies". EOS Trans. Am. Geophis. Union 75.

LI, J. e ZHANG, Y. (2006). "Prediction error method-based second order structural identification algorithm in stochastic state space formulation". Earthquake Engineering and Structural Dynamics. 35: 761-779. Published online in www.interscience.wiley.com. DOI 10.1002/eqe.563

LJUNG, L. (1999). "System Identification - Theory for the user". Prentice Hall, Upper Saddle River, NJ, $2^{\text {nd }}$ ed.

LJUNG, L. (2002). "Prediction Erro Estimation Methods". Circuits Systems Signal Processing. 21 (1): 11-21.

THE MATHWORKS INC. Matlab - "The Language of Technical Computing" - Version 7.0 (R14). 2004. Online Help.

MCT - Ministério da Ciência e Tecnologia. (2006). "Emissões de Dióxido de Carbono e Metano pelos Reservatórios Hidrelétricos Brasileiros". Primeiro Inventário Brasileiro de Emissões Antrópica de Gases de Efeito Estufa.

QINGCHANG, R. (1990). "Prediction Error Method for Identification of a Heat Exchanger". Eindhoven: Eindhoven University of Technology, Faculty of Electrical Engineering.

RUDD, J. W. M.; HARRIS, R.; KELLY, C. A. e HECKY, R. E. (1993). "Are hydroelectric reservoir significant sources of greenhouse gases?". Ambio 22: 246-248.

SODERSTROM, T., STOICA, P. FRIEDLANDER, B. (1991). "An Indirect Prediction Error Method for System identification". Automatica. 27 (1): 183-188.

SANTOS dos, M. A.; MATVIENKO. B.; ROSA, L. P.; SIKAR, E. (2005). "Carbon dioxide and methane emissions from hydroelectric reservoirs in Brasil". Proceedings of International Seminar on Greenhouse Fluxes from Hydro Reservoir and Workshop on Modeling Greenhouse Gas Emissions from Reservoir at Watershed Level. Rio de Janeiro. ELETROBRAS, COP- 
PE/UFRJ.

SBRISSIA, R.C. "Modelagem das espécies de carbono na coluna de água e predição de gases de efeito estufa em reservatórios. Estudo de caso: PCH Salto Natal, Campo Mourão, Paraná". Dissertação (Mestrado em Engenharia de Recursos Hídricos e Ambiental) - Setor de Tecnologia, Universidade Federal do Paraná. Curitiba, 2008

ST LLOUIS, V.L.; KELLY, C. A.; DUCHEMIN, E.; RUDD, J. W. M.; ROSENBERG, D. M. (2000). "Reservoirs surfaces as sources of greenhouse gases to atmosphere: A global estimate". BioScience 50: 766-755.

SUNG, S., LEE, I. (2001). "Prediction Error Identification Method for Continuous-Time Processes with Time Delay". Industrial Engineer Chemistry Research, 40: 5743-5751.

SVENSSON, B. (2005). "Greenhouse gas emission from hydroelectric reservoirs: a global perspective". Seminar on Greenhouse Fluxes from Hydro Reservoir and Workshop on Modeling Greenhouse Gas Emissions from Reservoir at Watershed Level. Rio de Janeiro. ELETROBRAS, COPPE/UFRJ

TUCCI, C.E.M. (2002). "Modelos Hidrológicos". Porto Alegre. ABRH.

Estimation of Greenhouse Gas Emissions in Reservoirs Based on the Dynamics of Organic Matter in the Water Column; Case Study of SHP Salto Natal, Campo Mourão, Paraná

\section{ABSTRACT}

In the 90's, research results suggested that some hydropower plant reservoirs could be a source of greenhouse gas emissions. Hence, to justify a new hydroelectric power plant project, it is necessary to show the best environmental solution, considering other energy sources. However, many studies for this purpose only consider monitoring greenhouse gases emissions, without taking into account the organic matter cycle in the reservoir and its relationship with the river basin. Thus, the goal of this research is to develop a methodology based on carbon mass balance to estimate methane $\left(\mathrm{CH}_{4}\right)$ and carbon dioxide $\left(\mathrm{CO}_{2}\right)$ fluxes from a small hydroelectric power plant reservoir, Salto Natal, located in Campo Mourão, Paraná, Brazil. In addition, it was also considered whether this reservoir is a carbon source or sink and also, whether it is from primary production or even if carbon sources come from outside the aquatic system (allochthonous) or from within the system itself (autochthonous). The results of the case study showed that it is an emitter of carbon dioxide with carbon sources from outside the reservoir (allochthonous) and that there is no methane flux.

Keywords: greenhouse gas emission, SHP reservoir, carbon balance. 